\title{
A Fast Analytic Model of Axial Flux Permanent Magnet Machines with Static/Dynamic Axis Eccentricity
}

\author{
Baocheng Guo and Yunkai Huang* \\ School of Electrical Engineering, Southeast University, Nanjing 210096, China
}

(Received 23 August 2016, Received in final form 6 December 2016, Accepted 6 December 2016)

\begin{abstract}
This paper presents a general analytical model to calculate the characteristics of axial-flux permanent-magnet machines with axis eccentricities. The radial and tangential magnetic flux densities in the air gap under normal conditions were first obtained using a combination of Maxwell's equations and Schwarz-Christoffel (SC) transformation. Next, equations for the radii were deduced to investigate the static/dynamic eccentricities. The back electromotive forces (EMFs) were calculated and compared with those obtained from finite element (FE) analysis. The analytical predictions show good agreement with the FE results. Detection approaches were obtained by comparing with normal conditions, and the analytical model was verified experimentally.
\end{abstract}

Keywords : analytical model, axial flux PM machines, back EMFs, eccentricities

\section{Introduction}

With benefits of compact construction and high power density [1], axial-flux permanent-magnet machines (AFPMMs) have been widely used in industrial applications such as electric vehicles (EV.), hybrid electric vehicles (HEVs) [2], and flywheel energy storage systems (FESSs) [3]. However, in manufacturing, the contact area between the rotor and shaft is relatively small, which predisposes it to eccentricities such as angular and misalignment eccentricities. Eccentricity can cause an unbalanced magnetic force [4] and mechanical vibration [5] during operation.

Various techniques can be used to model AFPMMs with eccentricity conditions, such as analytical models, magnetic equivalent circuits (MECs) [6], finite element (FE) analysis [7], or a combinations of these methods [8]. FE analysis can precisely model the influence of eccentricity by accurate computation of the air gap region. However, it is time-consuming because the eccentricity conditions of AFPMMs require a 3D model for the specific magnetic structures.

An alternative method is the field reconstruction (FR) method [9], which is a mix between analytical and FE

\footnotetext{
(C)The Korean Magnetics Society. All rights reserved.

*Corresponding author: Tel: +86-2583794169-807

Fax: +86-2583791696, e-mail: huangyk@seu.edu.cn;

guobaocheng1986@gmail.com
}

methods and partly depends on the FE model. Although it is much faster than $3 \mathrm{D}$ FE modeling, it still requires much time to model the different geometries. Analytical approaches are still regarded as effective and favorable methods due to their fast and acceptable results, and they are widely used among EM designers.

MEC is not accurate enough for some qualities like cogging torque and forces because of node limits. Also, the alignment of the rotor and stator necessitates the application of several simulation models [10]. In addition, there is more complexity when dealing with a non-uniform air gap. There are other possible analytical solutions $[11,12]$, but these methods focus on the static angular eccentricity. Moreover, the magnetic flux calculation in the air gap is not accurate enough.

To overcome these problems, Maxwell's equations and the Schwarz-Christoffel (SC) method are used in this paper to calculate the magnetic flux density of radial and tangential directions in the air gap. The classic SC model is called the conformal mapping (CM) method, which has a serious disadvantage in that it neglects the interaction between neighboring slots. The SC method can solve this problem to some extent, and it can be developed via the Matlab SC Toolbox [13].

This paper is organized as follows. In Section 2, a double rotor AFPMM with a concentrated coil is described provided. Section 3 introduces the electromagnetic calculations and SC transformations used in this paper. 
Axis eccentricities and their mathematic descriptions are presented in Section 4. The modeling of normal and eccentric magnetic distributions is introduced in Section 5 , and Section 6 discusses experimental results that verify the accuracy of the method. Lastly, conclusions are presented.

\section{Description of the AFPMM Prototype}

A quasi-3D-method was used to convert a 3D model to a $2 \mathrm{D}$ model to reduce the computation time. The machine is divided into a number of layers. The axial machine can be considered to be composed of several individual linear machines, and computations for each plane can be done separately.

Figure 1 shows the construction of the test machine and the principle of the quasi-3D method. SMC material is used for the stator core to improve the magnetic qualities $[14,15]$. The dimensions and specifications of the machine are shown in Table 1. A 2D model developed in 2D Cartesian coordinates is shown in Fig. 1. The average radius of the $i$-th layer is given by:

$$
\begin{gathered}
R_{\text {ave }}^{i}=R_{i}+\frac{R_{o}-R_{i}}{2 n_{s}}(2 i-1), \quad i=1,2, \ldots, n . \\
t_{c p}=\frac{R_{o}-R_{i}}{n_{s}}
\end{gathered}
$$

where $n_{s}$ is the number of slices, and $t_{c p}$ is the width of slice.

For AFPMMs with a constant ratio at the magnet length to the pole-pitch, using the mean radius only as a computation plane may produce proper results. However, in eccentricity conditions, the magnet length varies along the radial direction, so the results are not accurate since the magnetic characteristics are highly dependent on the

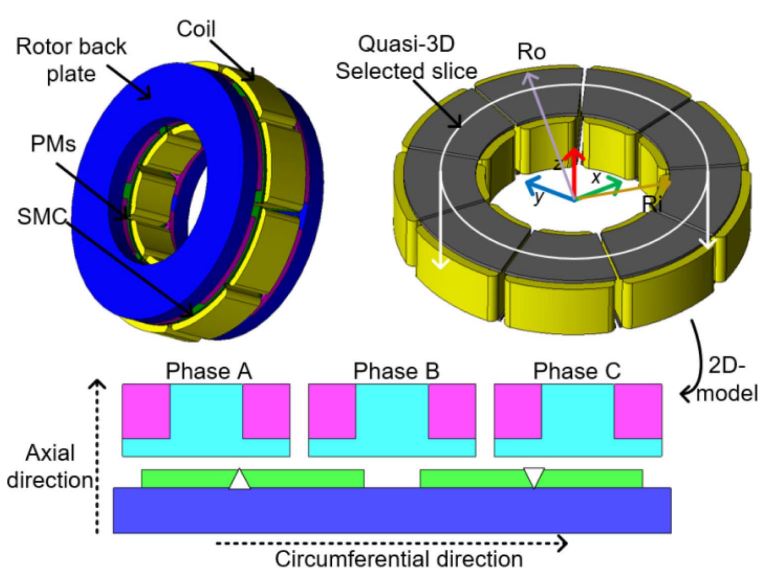

Fig. 1. (Color online) Construction of AFPMM (top) and the principle of quasi-3D method (bottom).
Table 1. Parameters of the 6-pole, 9-slot AFPMM.

\begin{tabular}{cccc}
\hline \hline Parameter & Symbol & Value & Unit \\
\hline Rated power & $\mathrm{P}$ & 10 & $\mathrm{~kW}$ \\
Rated voltage & $\mathrm{U}$ & 200 & $\mathrm{~V}$ \\
Rated speed & $\mathrm{n}_{\mathrm{p}}$ & 20,000 & $\mathrm{rpm}$ \\
Number of poles / slots & $\mathrm{p} / \mathrm{Q}_{\mathrm{s}}$ & $6 / 9$ & - \\
Stator outer radius & $\mathrm{R}_{\mathrm{o}}$ & 70 & $\mathrm{~mm}$ \\
Stator inner radius & $\mathrm{R}_{\mathrm{i}}$ & 45 & $\mathrm{~mm}$ \\
Air gap length & $\mathrm{g}_{0}$ & 3 & $\mathrm{~mm}$ \\
Remnant flux density of PM & $\mathrm{B}_{\mathrm{r}}$ & 1.03 & $\mathrm{~T}$ \\
Stator core & - & $\mathrm{SMC}$ & - \\
Permanent magnet & - & $\mathrm{NdFeB}$ & - \\
\hline
\end{tabular}

shape of the teeth and magnets. To improve the accuracy of the results, six slices are considered to calculate the normal and fault conditions.

\section{Calculation of Magnetic Field}

A previous approach [10] is used to calculate the magnetic induction in the air gap caused by the magnets. The following assumptions are made to reduce the complexity of the computations:

1) The magnetic material has uniform magnetization, and the relative recoil permeability $\mu_{r}$ is constant with a value close to unity, as in $\mathrm{NdFeB}$ materials.

2) Magnetic saturation is absent, and the rotor iron cores have infinite magnetic permeability.

3) Eddy current effects are neglected, which avoids the need for a complex eddy current field formulation.

\subsection{Model of PMs}

As shown in Fig. 2, the magnetization vector is assumed to be along the axial direction and can be described by a Fourier series:

$$
M_{n}=\frac{4 B_{r}}{n \pi \mu_{0}} \sin \left(\frac{n \pi \alpha_{p}}{2}\right)
$$

where $\alpha_{p}$ is the ratio of the magnet pole arc to the pole pitch. This results in two field components. The first is the air space (region I):

$$
\begin{aligned}
B_{x I}= & \mu_{0} H_{x I}=\mu_{0} M_{n} \sum_{n=1,3,5, \ldots}^{\infty} \sinh \left(\left(n \pi h_{m} / \tau_{p}\right) / \Delta\right) \\
& \times \sinh \left\{n \pi\left(g_{0}-y\right) / \tau_{p}\right\} \sin \left(n \pi x / \tau_{p}\right) \\
B_{y I}= & \mu_{0} H_{y I}=\mu_{0} M_{n} \sum_{n=1,3,5, \ldots \ldots}^{\infty} \sinh \left(\left(n \pi h_{m} / \tau_{p}\right) / \Delta\right) \\
& \times \cosh \left\{n \pi\left(g_{0}-y\right) / \tau_{p}\right\} \cos \left(n \pi x / \tau_{p}\right)
\end{aligned}
$$




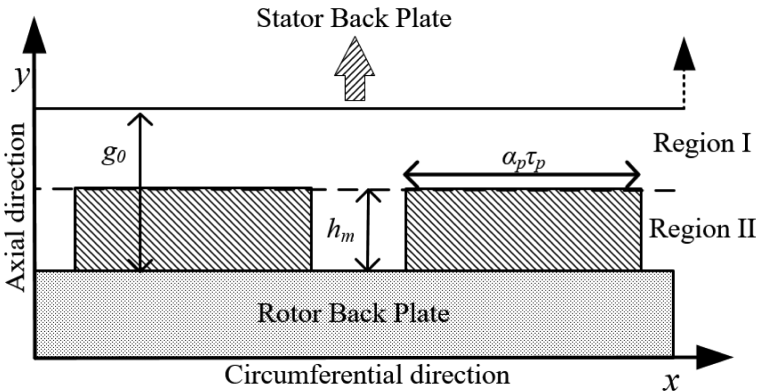

Fig. 2. Model of air gap flux caused by magnets.

where $t_{p}$ is the pole pitch in the circumferential direction, $h_{m}$ is the axial thickness of the magnets, $g$ is the axial distance between the rotor and stator back plates, and:

$$
\begin{aligned}
\Delta= & \mu_{r} \cosh \left(n \pi h_{m} / \tau_{p}\right) \sin \left\{n \pi\left(g_{0}-h_{m}\right) / \tau_{p}\right\} \\
& +\cos \left\{n \pi\left(g_{0}-h_{m}\right) / \tau_{p}\right\} \sinh \left(n \pi h_{m} / \tau_{p}\right)
\end{aligned}
$$

\subsection{Model of SC Mapping}

In the second step, the effect of stator slotting is included by using a numerical SC mapping technique [16]. The complex relative air gap permeance $\left(\lambda^{*}\right)$ is introduced to obtain the final magnet flux in the slot region:

$$
B_{z}=B_{t} \cdot \lambda^{*}=\left(B_{y}+j \cdot B_{x}\right) \cdot\left(\operatorname{Re}\left(\lambda^{*}\right)+j \cdot \operatorname{Im}\left(\lambda^{*}\right)\right)
$$

For AFPMMs, they can be calculated as:

$$
\lambda^{*}=\frac{\partial K}{\partial T} \cdot \frac{\partial T}{\partial Z} \cdot \frac{1}{\lambda_{0}}=\lambda_{y}+j \cdot \lambda_{x}
$$

where $\lambda_{0}$ is the complex permeance in the slotless air gap T-plane, while $\lambda_{x}$ and $\lambda_{y}$ are the tangential and radial components of the complex relative air gap permeance in the original Z-plane. $K, T$, and $Z$ represent the $K$-plane, $T$ plane, and $Z$-plane, respectively, as shown in Fig. 3.

The transformation is done in two steps. The complex plane (Z-plane) is first transformed to the rectangle domain ( $T$-plane), which is then transformed to the annular domain $(K$-plane). More detailed information is available elsewhere

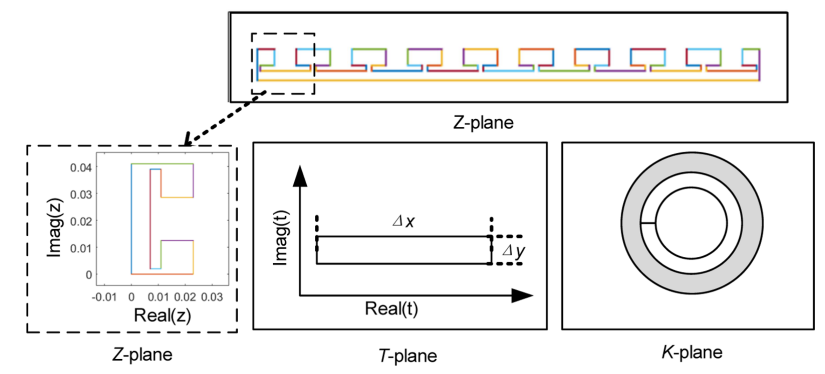

Fig. 3. (Color online) SC mapping on different planes.
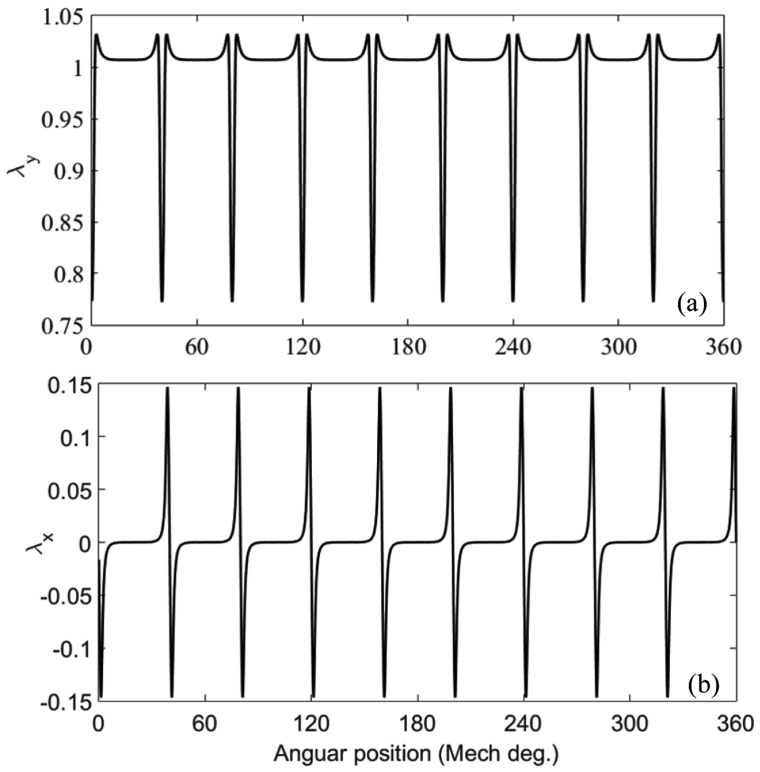

Fig. 4. Complex relative permeance without eccentricity in the middle of the air gap. (a) Radial component. (b) Tangential component.

[17]. Figure 4 shows the radial and tangential components of the relative permeance in the middle of the air gap.

\section{Eccentricities}

This section describes the static and dynamic axial eccentricities. The radii equations for calculating the back EMFs are also introduced.

\subsection{Axis Eccentricity Condition}

Static axial eccentricity occurs when the stator and rotor are offset from one another but the rotor is spinning around its own center, as shown in Fig. 5. The radius of eccentricity $R_{e c c s}$ at the angle $\beta_{s}$ is:

$$
\begin{aligned}
& R_{e c c}(\theta)=\sqrt{R_{m i d}^{2}+E_{c c s}^{2}-2 R_{m i d} E_{c c s} \cos \left(\pi-\theta_{s}\right)} \\
& \beta_{s}=\operatorname{acos}\left(\frac{R_{m i d}^{2}+R_{e c c}^{2}-E_{c c s}^{2}}{2 R_{\text {mid }} R_{e c c}}\right)
\end{aligned}
$$

where $E_{c c s}$ is the maximum offset distance, and $\theta_{s}$ is the location of the slot at angle $\theta$.

Using Eq. (8), the radius of the stator can be obtained based on the reference radius $\left(R_{\text {mid }}\right)$. The eccentricity radius can then be mapped to the corresponding radius of the rotor. We can change the ratio of the magnet pole arc to the pole pitch at each angle $\theta_{s}$ in (3) and (4) to calculate the flux density.

Dynamic axis eccentricity occurs when the rotor shaft is 


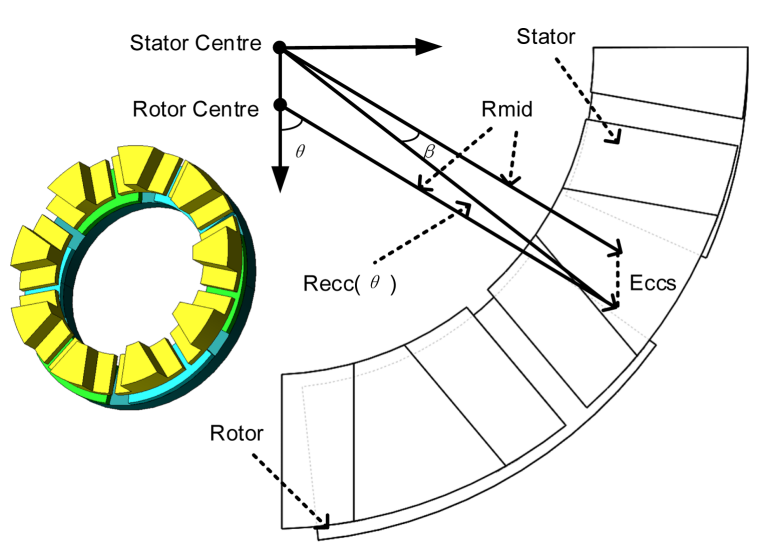

Fig. 5. (Color online) Configuration of the static axis eccentricity condition.

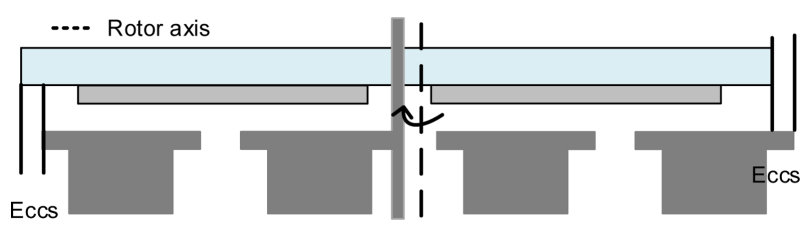

Fig. 6. (Color online) Configuration of the dynamic axis eccentricity condition.

offset from the rotor center and the rotor is spinning around the same center stator, as shown in Fig. 6. This eccentricity has similar characteristics in the rotating frame in that the pole has a constant eccentricity while the slot in the stator has a sinusoidal variation from the static reference frame.

A similar process is used to obtain the radius equation of the dynamic axis eccentricity, which can be described
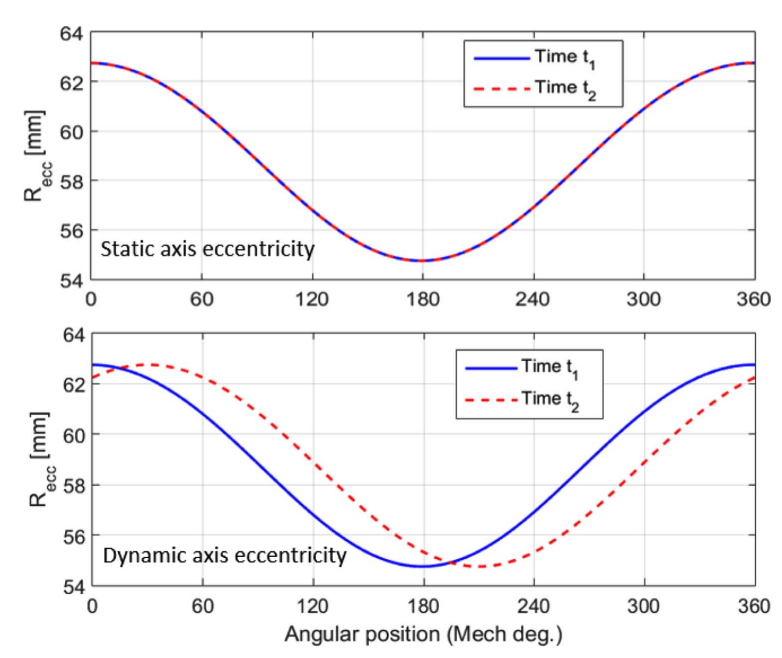

Fig. 7. (Color online) The length of $R_{e c c}$ under static/dynamic angular eccentricity. as:

$$
R_{e c c}(\theta)=\sqrt{R_{\text {mid }}^{2}+E_{c c s}^{2}-2 R_{\text {mid }} E_{c c s} \cos \left(\pi-\theta_{s}-\omega t\right)}
$$

The magnitude of $R_{\text {eccs }}$ at two simulation times under static/dynamic axis eccentricity $\left(E_{c c s}=4 \mathrm{~mm}\right)$ is shown in Fig. 7. The magnitude is unchanged under static eccentricities (for both angular and axial). Phase shift appears only under dynamic eccentricities.

\section{Results and Discussion}

To validate the analytical model, an axial flux permanent magnet machine with 9 slots and 6 poles was investigated, as shown in Table 1. The flux linkage is equal to the integral of the air gap flux density distribution across one coil pitch:

$$
\psi_{c, i}=\frac{R_{a v e, i}\left(R_{o}-R_{i}\right)}{n_{s}} \int_{\theta_{0}}^{\theta_{0}+\theta_{c}} B_{y, i} d \theta
$$

where $\theta_{0}$ is the coil starting side angle from the origin, $\theta_{c}$ is the expansion angle of the coil pitch, and $B_{y, i}$ is the axial component of the flux density in the $i_{\text {th }}$ layer. The prototype machine has two rotors and one stator, so the flux linkage of the $k_{t h}$ tooth coil with $N_{c}$ turns is simply given by:

$$
\psi_{k}=N_{c}\left(\psi_{c, i, L k}+\psi_{c, i, R k}\right) / 2
$$

The back EMF per phase is calculated by considering all coils of the phase winding to be connected in parallel. Based on the coil arrangement shown in Fig. 1, the back EMF in the $i_{t h}$ layer $E_{A, j}$ for phase A of the test machine is:

$$
E_{A, i}=-N_{c} \frac{R_{a v e, i}\left(R_{o}-R_{i}\right)}{n_{s}} \frac{d \psi_{k}}{d t}
$$

Based on the quasi-3-D consumption, the back EMF for the whole machines is calculated as:

$$
E_{A}=\sum_{i}^{N_{t}} E_{A, i}
$$

\subsection{Normal Conditions}

The radial and tangential components of the magnetic field at two time steps are shown in Fig. 8, which show good agreement between the analytical and FE model results. The back EMFs obtained from Eqs. (11)-(14) are shown in Fig. 9. Only the phase $A$ component is compared with the FE model, and this figure also shows good agreement between the results. 

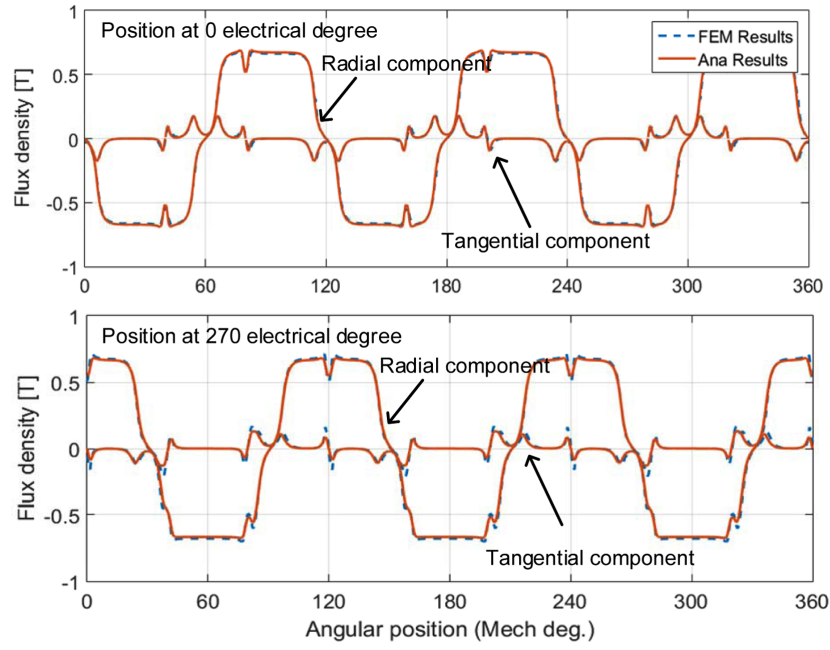

Fig. 8. (Color online) Radial and tangential components of flux density at the center of the air gap of the PM at two time steps.

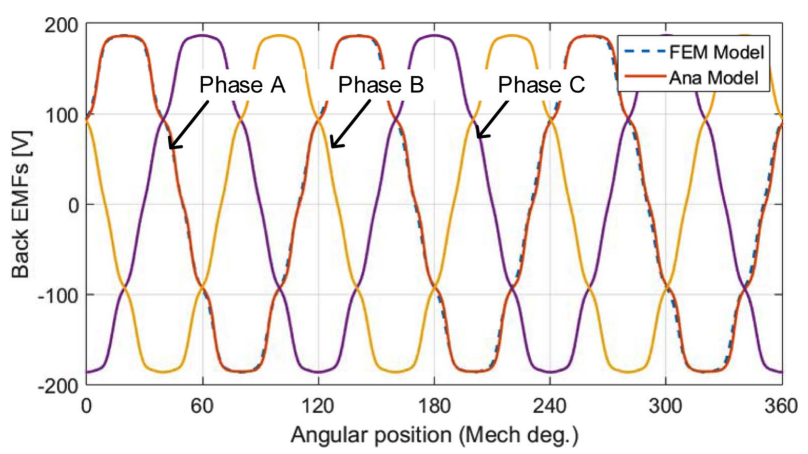

Fig. 9. (Color online) Back EMFs under normal condition.

\subsection{Axis Eccentricity Calculation}

To simulate the axis eccentricities, the parts of the poles overhanging the outer and inner radii of the stator are ignored, the maximum axis eccentricity $\left(E_{c c s}\right)$ is set as 4 $\mathrm{mm}$, and the initial angle $(\theta)$ shown in Fig. 5 is set as 0 degrees. In regard to the static/dynamic axis eccentricities, the changed radii of the eccentricity are introduced into Eqs. (4) and (5) along with the angle. The axis eccentricities could change the pole-arc ratio, and the period of the flux density wave would no longer be constant. Figure 10 presents the flux density under axis eccentricities at two time steps.

The calculated flux densities under eccentricities show good agreement with the results of the FE model. To illustrate the difference between the two eccentricity types, the flux densities were compared for when rotor mechanically rotates 180 degrees, as shown in Fig. 11. The results show that the periodic width of the flux density is not constant along the circumference direction, and the periodic width along the angular position for the static axis
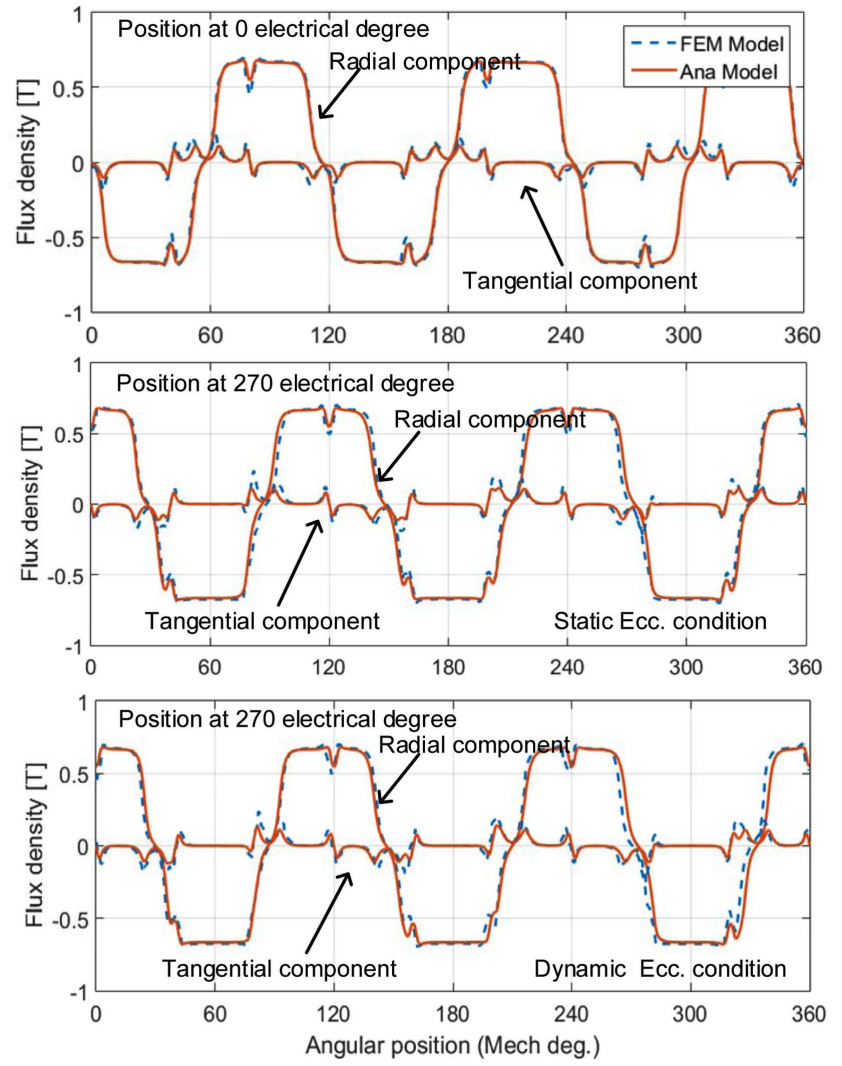

Fig. 10. (Color online) Radial and tangential components of the flux density at the air gap under static/dynamic axis eccentricities.

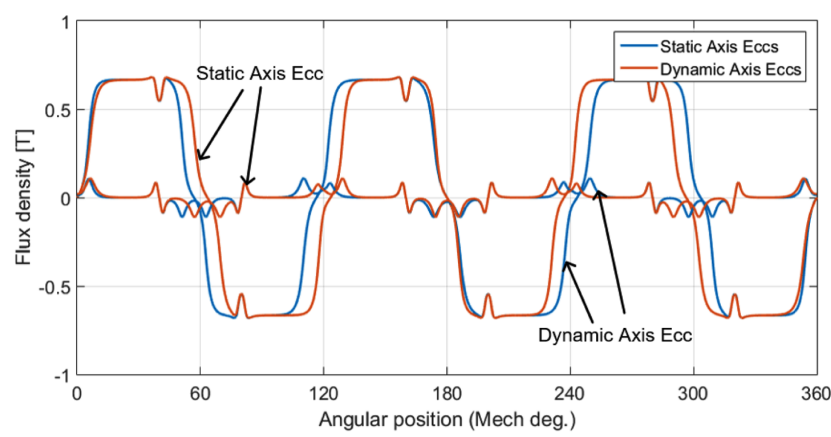

Fig. 11. (Color online) Radial and tangential components of the flux density at the air gap when rotating 180 degrees.

eccentricity does not change with time, but the dynamic eccentricity does.

The back EMFs resulting from static axis eccentricity are shown in Fig. 12. The amplitude of the phase $A$ EMF decreased, and the value is equal at each electrical period. This phenomenon can be simply explained by the polearc ratio, which decreases at the phase $A$ position. In addition, it does not change with time. For comparison with the back EMFs of the static axis eccentricity, Fig. 13 shows the results for dynamic axis eccentricity. The 


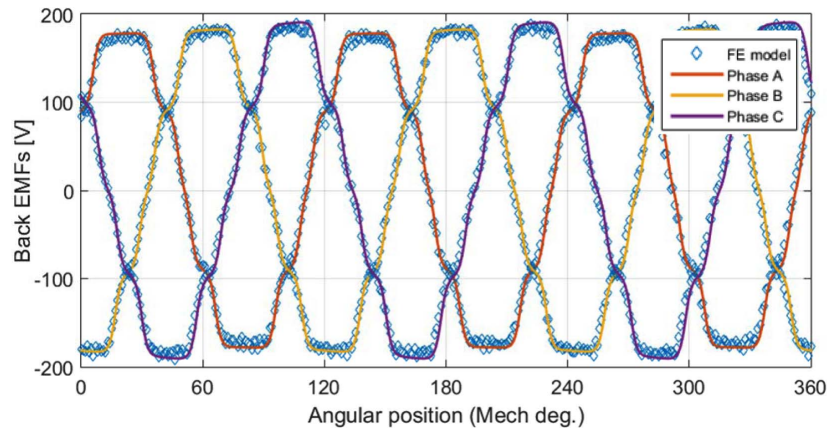

Fig. 12. (Color online) Back EMFs under static axis eccentricity.

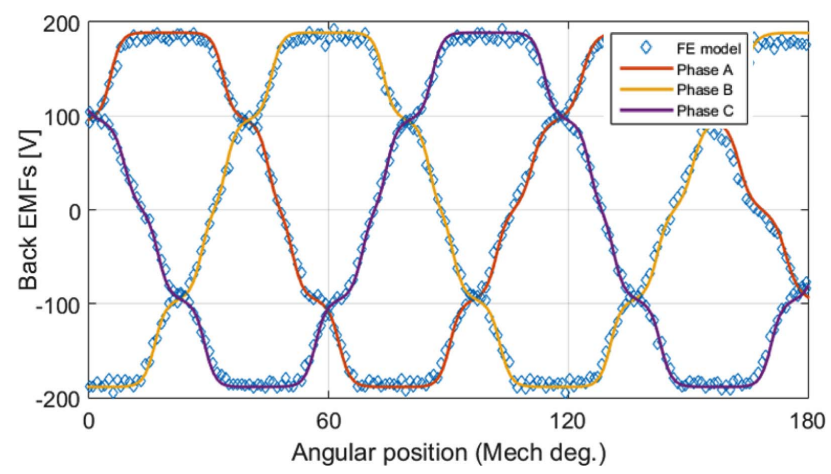

Fig. 13. (Color online) Back EMFs under dynamic axis eccentricity.

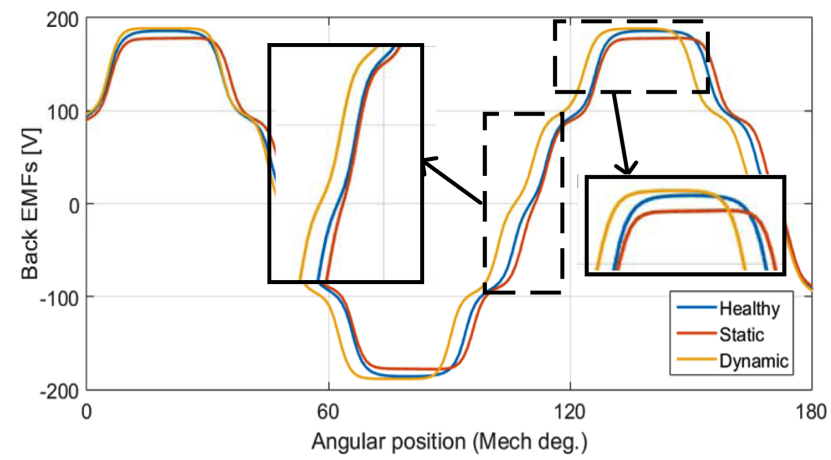

Fig. 14. (Color online) Back EMFs under dynamic axis eccentricity.

proposed method shows good agreement with the FE model.

Figure 14 shows the difference of the back EMFs between normal and static/dynamic axis eccentricity conditions. The back EMF of the dynamic model has a relatively large angle shift in comparison to that for the static eccentricity. The static eccentricity offset position can be found easily by detecting the amplitude, but it is difficult to detect dynamic eccentricity.
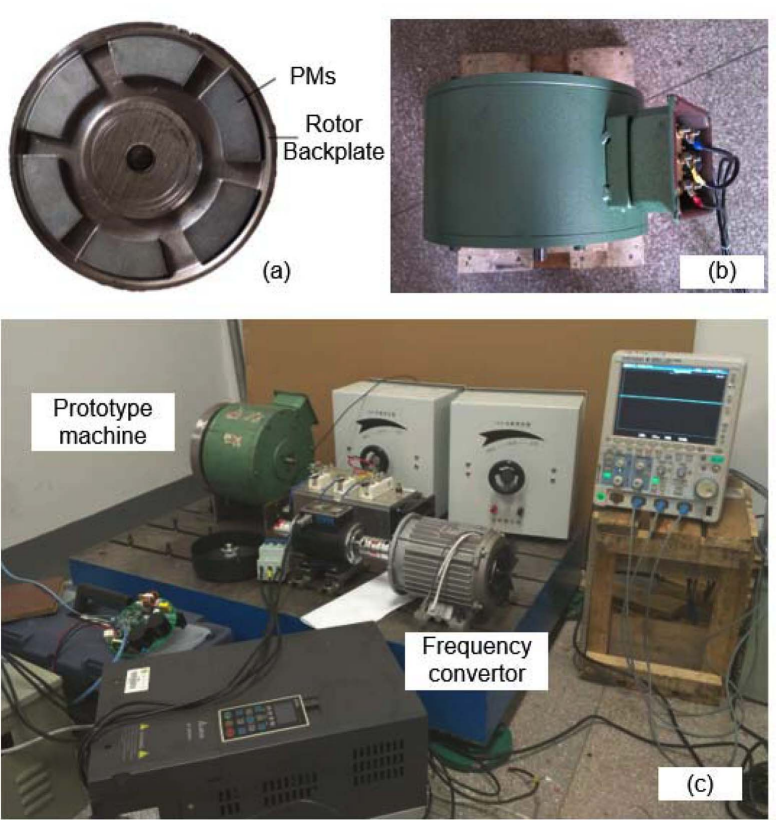

Fig. 15. (Color online) Experimental test rig.

\section{Experimental Results}

The rotor disk and the double rotor AFPMM prototype used in the experiment are shown in Figs. 15 (a) and (b), respectively. The experimental setup and devices are shown in Fig. 21 (c). The AFPM motor was driven by a frequency converter to test the motor performance, and the no-load parameters such as the back EMF were tested using a separate driving motor and a belt.

For the experiment, a no-load test was done at $300 \mathrm{rpm}$, and the back EMF was measured, as shown in Fig. 15. The results are highly similar to the analytical results in Fig. 16. Table 2 shows the back EMF coefficients obtain-

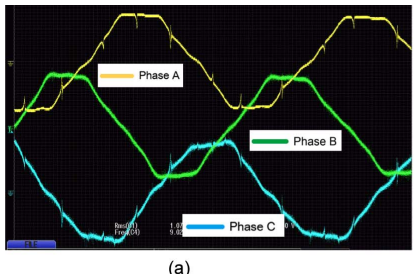

Fig. 16. (Color online) Back EMF results.

Table 2. Back EMF coefficient obtained by different methods.

\begin{tabular}{cccc}
\hline \hline & FE model & Analytical model & Experiment \\
\hline Back EMF Coeff. & 5.30 & 5.30 & 5.28 \\
Errors & $0.37 \%$ & $0.37 \%$ & \\
\hline
\end{tabular}


ed by the analytical model, the FE model, and the experiment. There is a slight RMS error between the analytical results and the real values.

The analytical model requires only 206 seconds of computation to obtain the cogging torque and electromagnetic torque with one mechanical cycle with eccentricities. However, the 3D FE model requires almost 27 hours. Although there is similar error when comparing the analytical results to the experimental values, the amount is acceptable. Thus, the proposed approach could save time and achieve acceptable results.

\section{Conclusion}

This paper has presented an approach for modeling AFPMMs with stator/dynamic axis eccentricities. A quasi3D method was used to account for the 3D intrinsic nature of the AFPM machine. The back EMFs of the AFPM machine under normal and eccentricity conditions were calculated, and the accuracy of the method was verified using a 3D FE model. The axis eccentricity conditions could be detected by analyzing the amplitude and phase of the back EMFs. The computation time was reduced remarkably while maintaining high accuracy at the same level as the FE model. Finally, an experiment carried verified the accuracy of the proposed method.

\section{Acknowledgments}

This work was supported in part by the National Nature Science Foundation of China (Project Number 51377019), the Scientific and Technological Innovation Program of Jiangsu Province (Project Number BY201412715), and the Qing Lan Project.

\section{References}

[1] J. Lee, B. Park, and B. Woo, J. Magn. 20, 413 (2015).

[2] M. Polikarpova, P. Ponomarev, P. Lindh, I. Petrov, W. Jara, V. Naumanen, J. A. Tapia, and J. Pyrhonen, IEEE Trans. Ind. Electron. 62, 7382 (2015).

[3] T. D. Nguyen, K.-J. Tseng, S. Zhang, and T. D. Nguyen, IEEE Trans. Ind. Electron. 58, 3784 (2011).

[4] S. M. Mirimani, A. Vahedi, and F. Marignetti, IEEE Trans. Magn. 48, 143 (2012).

[5] J. Li, R. Qu, and Y.-H. Cho, 2014 International Conference on Electrical Machines (ICEM), 502 (2014).

[6] K. Abbaszadeh and S. S. Maroufian, 2013 21st Iranian Conference on Electrical Engineering (ICEE), 1 (2013).

[7] S. M. Mirimani, A. Vahedi, F. Marignetti, and E. De Santis, IEEE Trans. Ind. Appl. 48, 1838 (2012).

[8] E. Ajily, M. Ardebili, and K. Abbaszadeh, IEEE Trans. Energy Convers. 31, 486 (2015).

[9] E. Ajily, K. Abbaszadeh, and M. Ardebili, IEEE Trans. Energy Convers. 30, 199 (2015).

[10] A. Hemeida and P. Sergeant, IEEE Trans. Magn. 50, 1 (2014.

[11] K. Abbaszadeh and A. Rahimi, Sci. Iran. Trans. Comput. Sci. Eng. Electr. 22, 2482 (2015).

[12] F. Marignetti, A. Vahedi, and S. M. Mirimani, Electr. Power Compon. Syst. 43, 1039 (2015).

[13] E. Ilhan, E. T. Motoasca, J. J. Paulides, and E. A. Lomonova, Math. Sci. 6, 1 (2012).

[14] Y. G. Guo and J. G. Zhu, Aust. J. Electr. Electron. Eng. 3, 37 (2006).

[15] Y. Huang, J. Zhu, Y. Guo, Z. Lin, and Q. Hu, IEEE Trans. Magn. 43, 2492 (2007).

[16] D. Zarko, D. Ban, and T. A. Lipo, IEEE Trans. Magn. 42, 1828 (2006).

[17] T. A. Driscoll and L. N. Trefethen, Schwarz-Christoffel mapping. Cambridge?; New York: Cambridge University Press (2002). 